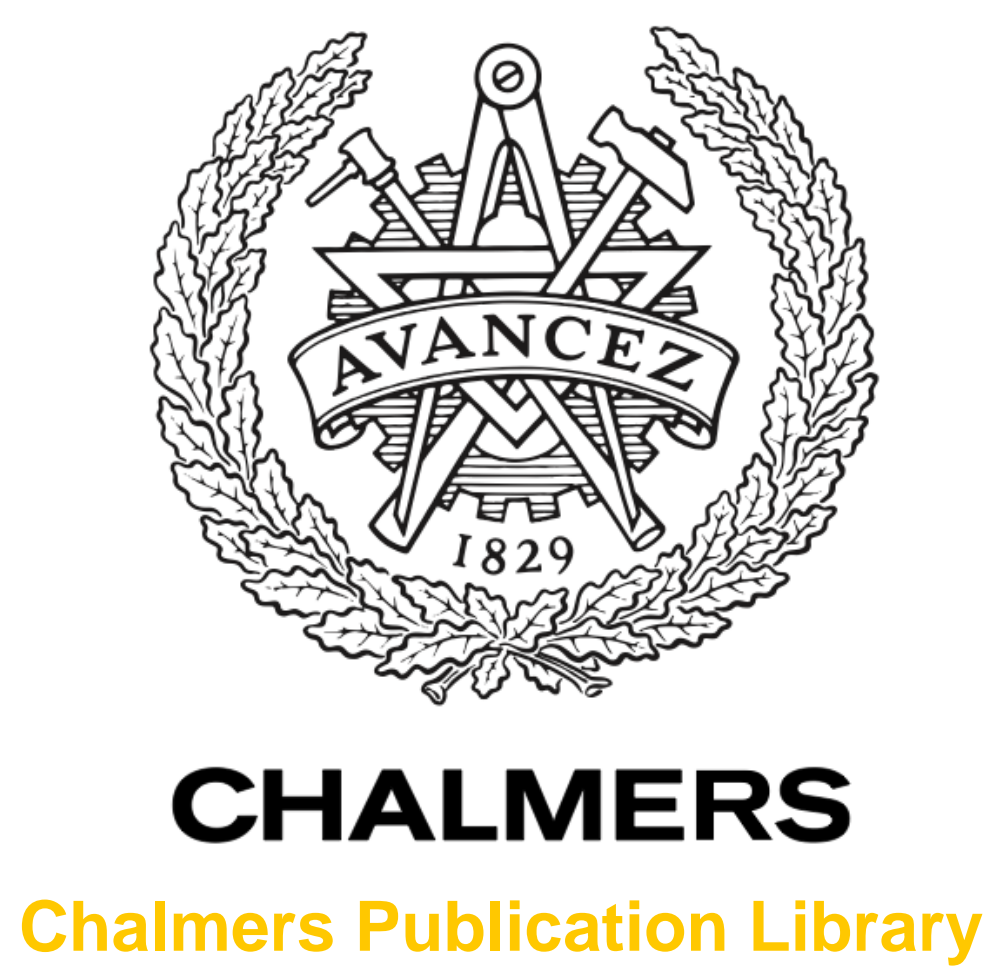

Frequency and polarization switched QPSK

This document has been downloaded from Chalmers Publication Library (CPL). It is the author's version of a work that was accepted for publication in:

Proc. European Conference on Optical Communication (ECOC)

Citation for the published paper:

Eriksson, T. ; Johannisson, P. ; Sjödin, M. (2013) "Frequency and polarization switched QPSK". Proc. European Conference on Optical Communication (ECOC)

Downloaded from: http://publications.lib.chalmers.se/publication/192132

Notice: Changes introduced as a result of publishing processes such as copy-editing and formatting may not be reflected in this document. For a definitive version of this work, please refer to the published source. Please note that access to the published version might require a subscription. 


\title{
Frequency and Polarization Switched QPSK
}

\author{
Tobias A. Eriksson ${ }^{(1)}$, Pontus Johannisson ${ }^{(1)}$, Martin Sjödin ${ }^{(1)}$, Erik Agrell ${ }^{(2)}$, \\ Peter A. Andrekson ${ }^{(1)}$, Magnus Karlsson ${ }^{(1)}$ \\ (1) Department of Microtechnology and Nanoscience. ${ }^{(2)}$ Department of Signals and Systems. \\ Chalmers University of Technology, SE-412 96, Gothenburg, Sweden. Email: tobias.eriksson@chalmers.se
}

\begin{abstract}
We propose 8-dimensional biorthogonal modulation as a format with $3 \mathrm{~dB}$ increased asymptotic power efficiency over PM-QPSK. We demonstrate one possible experimental implementation of this format based on frequency and polarization switching and compare with dual-carrier PM-QPSK and PS-QPSK.
\end{abstract}

\section{Introduction}

In recent years, there has been an increased research interest in power efficient modulation formats much due to the publications by Bülow ${ }^{1}$, as well as Agrell and Karlsson ${ }^{2,3}$. Modulation formats that are optimized in the 4-dimensional (4D) space spanned by the in-phase and quadrature components for the two polarization states of the optical field can achieve a higher power efficiency than standard polarization multiplexed (PM) 2-dimensional (2D) formats since the minimum Euclidean distance between symbols can be increased by optimization in a higher-dimension space. Examples of such 4D modulation formats are polarization-switched QPSK (PS-QPSK) $)^{2,4,5}$, 6-ary polarization-shift keying (6PolSK) $)^{1,6}$ and 128-ary set-partioned QAM $(128-S P-Q A M)^{7,8}$.

In this paper, we explore the possibility to increase the dimensionality of an optical signal to 8 dimensions by treating two closely spaced optical wavelengths as one signal, i.e. twice the 4D space of an optical field. This opens new possibilities to optimize modulation formats. We propose and investigate 4-ary frequency and polarization switched QPSK (4FPS-QPSK), which is an optimized 8-dimensional (8D) format, and compare this to dual-carrier (DC) PM-QPSK (DC-PM-QPSK) and DC-PS-QPSK at 10 Gbaud.

\section{FPS-QPSK}

Modulation in 8 dimensions can be achieved by using for instance binary pulse position modulation or as in this paper, by using two optical carriers as illustrated in Fig. 1. We propose the use of 8-dimensional biorthogonal modulation ${ }^{9}$ in the optical domain where the symbols are given by all permutations of $\left\{ \pm 1,0^{7}\right\}$. This can be realized by sending one QPSK symbol in one of the four possible choices of frequency and polarization. Hence, 2 bits are encoded in the QPSK symbol and 2 bits in the frequency and polarization switching. Thus, we call this format 4FPS-QPSK. The asymptotic power efficiency ${ }^{2}$ for any modulation format is given by $\gamma=d_{\min }^{2} \log _{2} M /\left(4 E_{s}\right)$, where $M$ is the number of symbols, $d_{\min }$ the minimum Euclidean distance, and $E_{s}$ the average energy per symbol ${ }^{2}$. The

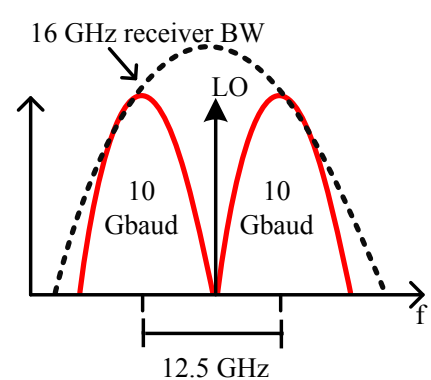

Figure 1. Illustration of the carrier spacing, the position of the local oscillator, and the electrical bandwidth of the receiver.

factor $1 / 4$ normalizes the expression so that $\gamma=0 \mathrm{~dB}$ for PM-QPSK. PS-QPSK has $\gamma=1.76 \mathrm{~dB}$ and 4FPSQPSK has $\gamma=3 \mathrm{~dB}$. Note that $\gamma$ is defined for the same bit rate. We are not aware of any $8 \mathrm{D}$ modulation format that is more power efficient.

In this paper we have chosen to compare 4FPSQPSK to DC-PM-QPSK and DC-PS-QPSK at the same symbol rate $(10 \mathrm{Gbaud})$, where we detect the two carriers using the same receiver as illustrated in Fig. 1. With the dual-carrier setup all three formats will occupy the same bandwidth. This means that 4FPS-QPSK carries 4 bit/symbol and has a bit rate of $40 \mathrm{Gbit} / \mathrm{s}$, DC-PS-QPSK $6 \mathrm{bit} / \mathrm{symbol}(60 \mathrm{Gbit} / \mathrm{s})$ and DC-PM-QPSK $8 \mathrm{bit} / \mathrm{symbol}(80 \mathrm{Gbit} / \mathrm{s})$. At the same symbol rate, the asymptotic sensitivity gain over DCPM-QPSK will thus be $3 \mathrm{~dB}$ for DC-PS-QPSK and $6 \mathrm{~dB}$ for 4FPS-QPSK.

\section{Experimental Setup}

The experimental setup is shown in Fig. 2. The transmitters for all modulation formats use two free-running distributed feedback lasers (DFB) at $\sim 1550 \mathrm{~nm}$ with $12.5 \mathrm{GHz}$ spacing and $\sim 100 \mathrm{kHz}$ linewidth. All pulse pattern generators (PPG) are clocked using a $10 \mathrm{GHz}$ frequency generator. To generate 10 Gbaud DC-PM-QPSK, each of the two lasers was followed by an I/Q-modulator to modulate QPSK symbols. Polarization multiplexing is then emulated by combining the two signals before split and recombination with orthogonal polarizations and with a temporal delay to decorrelate the data. To generate DC-PS-QPSK the two carriers are modulated 


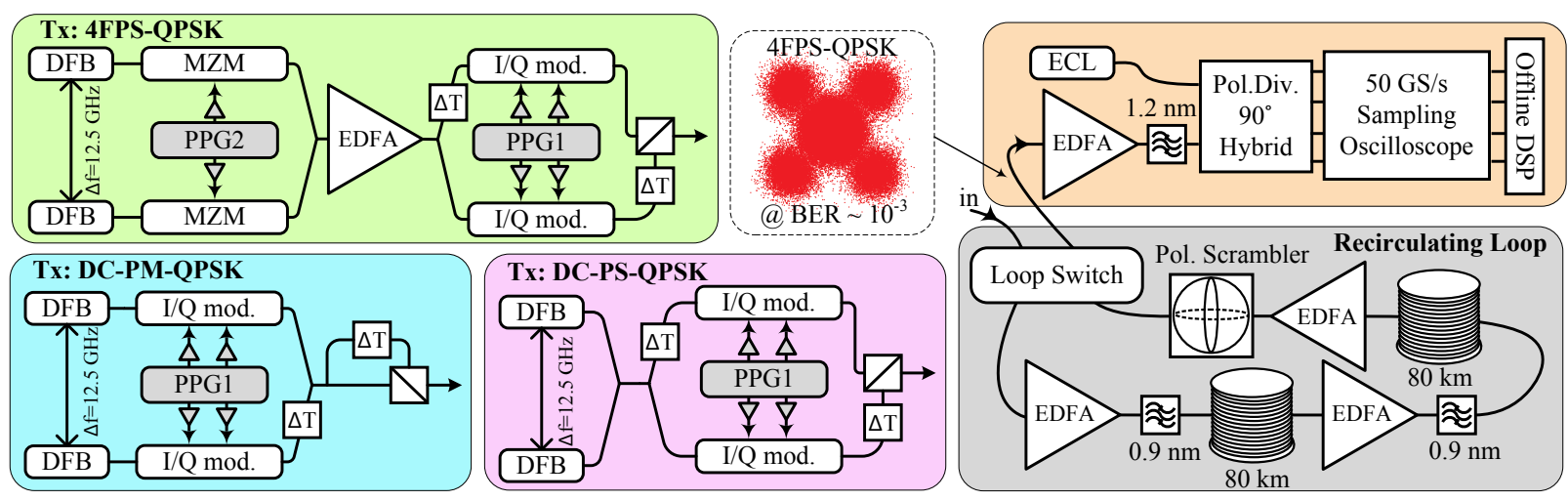

Figure 2. Experimental setup showing transmitters for 4FPS-QPSK, DC-PM-QPSK and DC-PS-QPSK. The MZMs in the 4FPS-QPSK transmitter are driven in a push-pull configuration. Recirculating loop with $2 \times 80 \mathrm{~km}$ spans of SSMF and a loop synchronized polarization scrambler. The signals are detected by a polarization diverse coherent receiver and sampled with a real-time sampling oscilloscope.

with one I/Q-modulator in each polarization state. PS-QPSK is generated by encoding one of the four bit-streams as an XOR-operation on the other three. In the 10 Gbaud 4FPS-QPSK transmitter, frequency shift keying (FSK) is generated by two Mach-Zehnder modulators (MZMs) driven in a push-pull configuration to select the carrier from only one laser at the time in each symbol slot. The FSK stage is followed by PS-QPSK generation. An erbium-doped fiber amplifier (EDFA) was placed in-between the FSK and PS-QPSK stages to ensure high OSNR after the 4FPS-QPSK transmitter. To synchronize the FSK patterns with the PS-QPSK generation as well as synchronizing the two polarization arms, two optical delay lines were used as seen in Fig. 2 in combination with delays in the electrical domain.

The signals from the three different transmitters were propagated through a recirculating loop with two spans of $80 \mathrm{~km} \mathrm{SSMF}$. Each span is preceded by a 0.9 $\mathrm{nm}$ optical bandpass filter to suppress the amplified spontaneous emission (ASE) noise. To compensate for the attenuation of each span, the signal is amplified by $5 \mathrm{~dB}$ noise figure EDFAs. A loop-synchronized polarization scrambler is used to avoid unrealistic accumulation of polarization impairments. A third EDFA is used in the loop to compensate for the loss in the polarization scrambler and the loop switching components. The signal is detected using a polarization diverse $90^{\circ}$ hybrid with integrated balanced photo receivers. $\mathrm{A} \sim 300 \mathrm{kHz}$ external cavity laser (ECL) is used as a local oscillator (LO). The frequency of the $\mathrm{LO}$ is adjusted to be centered between the two carriers, i.e. spaced $6.25 \mathrm{GHz}$ from each carrier. In this way, both carriers are detected simultaneously and sampled using a 50 Gsample/second real-time oscilloscope with $16 \mathrm{GHz}$ electrical bandwidth.

The digital signal processing (DSP) starts with two first order Gaussian bandpass filters with center frequencies matching the two carriers. After the filtering, the DSP is parallel for the two carriers. Optical front-end compensation is performed followed by an approximate frequency down-conversion of 6.25 GHz. Adaptive equalization and polarization demultiplexing is achieved by four 14-tap FIR-filters optimized using the constant modulus algorithm (CMA) for DC-PM-QPSK and PS-CMA ${ }^{10}$ for DCPS-QPSK. For 4FPS-QPSK we use a modified version of PS-CMA ${ }^{10}$ with a power threshold which estimates if a PS-QPSK symbol was sent or not. For the case below threshold, the taps are not updated. This equalizer required a smaller step-size and longer convergence time compared to PS-CMA. The equalizer is followed by frequency offset estimation based on the Fourier transform and phase estimation based on the Viterbi-Viterbi algorithm with a block length of 32 samples for DC-PS-QPSK and DC-PM-QPSK. For 4FPS-QPSK a longer block length of 121-171 samples, depending on launch power and transmission distance, had to be used since the FSK complicates the phase tracking due to the fact that the laser to be tracked is switched on and off. To decode the FSK bits of 4FPS-QPSK, the total power over both polarizations are compared at the two frequencies after the DSP. Finally, the bit-error rate (BER) is evaluated.

\section{Experimental Results}

We investigated the penalty from detecting two carriers using the same receiver in a dual-carrier setup with the LO spaced $6.25 \mathrm{GHz}$ from the signal laser, as illustrated in Fig. 1. We observe no extra implementation penalty, compared to conventional intradyne detection, when only one PM-QPSK channel is present. However, when we turn on the second channel, we observe a $0.3 \mathrm{~dB}$ extra crosstalk penalty.

In Fig. 3a, the back-to-back (B2B) performance as well as the theoretical performance ${ }^{9}$ for DC-PMQPSK, DC-PS-QPSK, and 4FPS-QPSK are shown. Note that we measured OSNR $(0.1 \mathrm{~nm})$ as the signal power including both carriers. At BER $=10^{-3}$, the implementation penalty for 4FPS-QPSK is $1.3 \mathrm{~dB}$ whereas for both DC-PM-QPSK and DC-PS-QPSK it is $0.9 \mathrm{~dB}$. The relative sensitivity gain is $4.3 \mathrm{~dB}$ for 


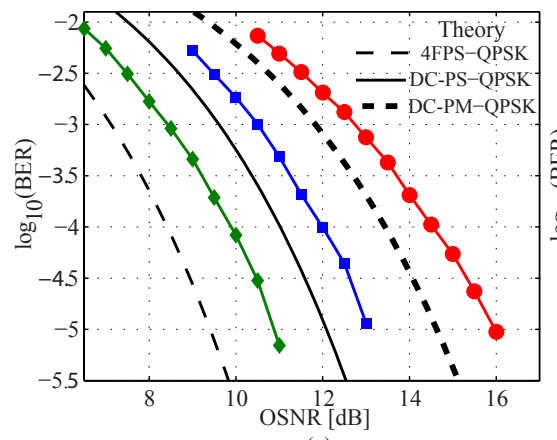

(a)

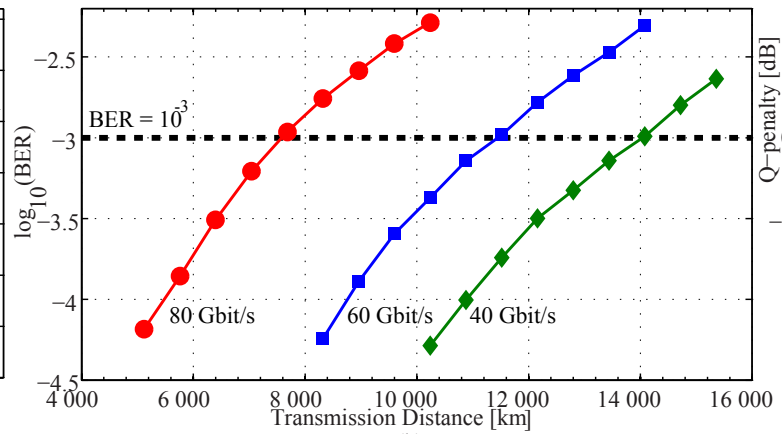

(b)

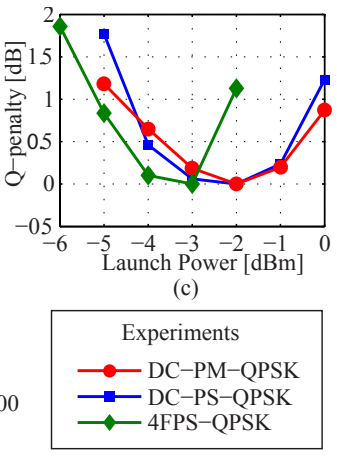

Experiments

- DC-PM-QPSK 4FPS-QPSK

Figure 3. (a) B2B performance and theoretical performance ${ }^{9}$ for 4 FPS-QPSK, DC-PS-QPSK and DC-PM-QPSK. OSNR is measured including signal power in both carriers. (b) Transmission results for the optimal launch power for each format. (c) Q-factor penalty as a function of launch power for each format. The Q-penalty is measured at a transmission distance for each format where $\mathrm{BER} \approx 10^{-3}$.

4FPS-QPSK over DC-PM-QPSK which is in good agreement with the $4.7 \mathrm{~dB}$ expected from theoretical predictions ${ }^{9}$. DC-PS-QPSK has a $2.2 \mathrm{~dB}$ sensitivity gain over DC-PM-QPSK and 4FPS-QPSK has a $2.1 \mathrm{~dB}$ sensitivity gain over DC-PS-QPSK.

The Q-factor penalties for different launch powers for all formats are seen in Fig. 3c. Note that the launch power was measured over both carriers. The optimal launch power is $-2 \mathrm{dBm}$ for both DC-PS-QPSK and DC-PM-QPSK whereas for 4FPS-QPSK it is $-3 \mathrm{dBm}$. It should be noted that 4FPS-QPSK suffers a high penalty at $-2 \mathrm{dBm}$ due to difficulties with phase tracking in combination with nonlinear phase-noise.

The transmission results for the optimal launch power for each format are shown in Fig. 3b. At a $\mathrm{BER}=10^{-3}$, we were able to transmit DC-PM-QPSK up to $7,600 \mathrm{~km}$, DC-PS-QPSK up to $11,400 \mathrm{~km}$ and 4FPS-QPSK up to $14,000 \mathrm{~km}$. This corresponds to an increase of $50 \%$ for DC-PS-QPSK over PM-QPSK and an increase of $84 \%$ for 4FPS-QPSK over DCPM-QPSK.

Clearly the gain in transmission reach for 4FPSQPSK is not as large as the B2B results indicate. One main factor that degrades the performance for 4FPSQPSK is a problem with the phase tracking due to the FSK modulation. Since on average $50 \%$ of the symbols in one channel are zero due to the FSK and the fact that many zeros in a row complicates the phase tracking further, a longer block length is needed for the phase tracking compared to PS-QPSK and PMQPSK. B2B, this only has a minor impact on the performance. However, at long transmission distances and for higher launch power this problem becomes extra prominent. The nonlinear phase-noise degrades the phase tracking performance even further, thus leading to considerable problems with cycle slips. We had to increase the block length over 4 times compared to DC-PM-QPSK to avoid a substantial amount of cycle slips and this does degrade the performance of the QPSK symbols in the 4FPS-QPSK modulation after transmission. This can be seen in Fig. 3c where we for a launch power of $-2 \mathrm{dBm}$ see a consider- able penalty from the longer block length. It should be noted that even with a longer block length, we still have a problem with cycle slips after long transmission when the OSNR is low. A possible solution to this would be to use phase locked light sources, by for instance using an optical comb generator in the transmitter instead of two free-running lasers. It would then be possible to perform a joint phase estimation for the two carriers. The adaptive equalizer used for 4FPS-QPSK is using decisions within the optimization of the taps which might be suboptimal, so further research on an equalizer for 4FPS-QPSK could possibly give better results. It is also possible to implement this format as a 2-ary pulse position modulation instead of using FSK, which also would solve the phase tracking problem. If the phase tracking can be optimized it is likely that the optimal launch power for 4FPS-QPSK would be increased which possibly would lead to an increase in transmission reach.

\section{Conclusions}

We have introduced a novel 8-dimensional modulation format, 4FPS-QPSK, which is an implementation of 16-ary biorthogonal modulation and in theory has a $3 \mathrm{~dB}$ asymptotic power efficiency gain over PMQPSK for the same bit rate and $6 \mathrm{~dB}$ over DC-PMQPSK at the same symbol rate. We experimentally demonstrated 10 Gbaud 4FPS-QPSK (40 Gbit/s) and showed a $4.2 \mathrm{~dB}$ B2B sensitivity gain (at BER $=10^{-3}$ ) over DC-PM-QPSK (80 Gbit/s) at the same symbol rate. We were able to transmit FPS-QPSK up to $14,000 \mathrm{~km}$ which corresponds to an $84 \%$ increase in transmission reach over DC-PM-QPSK.

\section{References}

[1] H. Bülow et al., Proc. OFC'09, OWG2 (2009).

[2] E. Agrell \& M. Karlsson, J. Lightw. Tech. 27, 5115 (2009).

[3] M. Karlsson \& E. Agrell, Opt. Exp. 17, 10814 (2009).

[4] M. Sjödin et al., Opt. Exp. 19, 7839 (2011).

[5] M. Nölle et al., Opt. Exp. 19, 24370 (2011).

[6] J. K. Fischer et al., Proc. ECOC'12, We.1.C.4 (2012).

[7] T. A. Eriksson et al., Proc. OFC'13, OTu3B.2 (2013).

[8] J. Renaudier et al., Proc. OFC'13, OTu3B.1 (2013).

[9] M. K. Simon et al., Digital Communication Techniques: Signal Design and Detection, Prentice Hall (1995).

[10] P. Johannisson et al., Opt. Exp. 19, 7734 (2011). 